\title{
Out-of-Pocket, Catastrophic Health Expenditure and Distress Financing on Non-Communicable Diseases in India: A Systematic Review with Meta-Analysis
}

\author{
Anushikha Dhankhar, Ranjeeta Kumari*, Yogesh A Bahurupi
}

\begin{abstract}
Objective: The aim of this systematic review is to determine pooled estimates of out-of-pocket (OOPE) and catastrophic health expenditure (CHE), correlates of CHE, and most common modes of distress financing on the treatment of selected non-communicable disease (cancer) among adults in India. Methods: PubMed, Scopus and Embase were searched for eligible studies using strict inclusion and exclusion criteria. Data was extracted and pooled estimates using random effects model of meta-analysis were determined for different types of costs. Forest plots were created and heterogeneity among studies was checked. Results: The pooled estimate of direct OOPE on inpatient and outpatient cancer care were 83396.07 INR (4405.96 USD) $(95 \%$ CI $=44591.05-122202.0)$ and $2653.12(140.17$ USD) INR (95\% CI $=-251.28-5557.53$ ), respectively, total direct OOPE was 47138.95 INR (2490.43 USD) (95\% CI $=37589.43-56690.74)$, indirect OOPE was $11908.50 \mathrm{INR}(629.15 \mathrm{USD})(95 \% \mathrm{CI}=-5909.33-29726.31)$ and proportion of individuals facing CHE was $62.7 \%$. However, high heterogeneity was observed among the studies. Savings, income, borrowing money and sale of assets were the most common modes of distress financing for cancer treatment. Conclusion: Income- and treatment-related cancer policies are needed to address the evidently high and unaffordable cancer treatment cost. Economic studies are needed for estimating all types of costs using standardised definitions and tools for precise estimates. Robust cancer database/registries and programs focusing on affordable cancer care can reduce the economic burden and prevent impoverishment.
\end{abstract}

Keywords: Out-of-pocket expenditure- catastrophic health expenditure- distress financing- NCDs- cancer- India

Asian Pac J Cancer Prev, 22 (3), 671-680

\section{Introduction}

Non-communicable diseases (NCDs) are long duration (chronic) diseases, which result from a combination of factors, such as physiology, genetics, lifestyle-related behaviour and environment (WHO, 2018). Annually, 71\% (41 million) of all global deaths are attributed to them (WHO, 2018). Among all, cancer has been reported to be the second leading cause of mortality globally, accounting for an estimated 9.6 million global deaths in the year 2018 (WHO, 2019). Cancer-related deaths range from $11.6 \%$ to $67.2 \%$ in India (Nethan et al., 2017).

In India, the total number of most common cancers diagnosed in the year 2018 was 168,122 which includes oral, breast and cervical cancer (CBHI, 2019). Owing to its chronic nature and expensive treatment, out-of-pocket expenditure (OOPE) is the highest for cancer. In India, the insurance coverage is limited and health expenditures are not covered for approximately $80 \%$ of the population (Government of India, NSSO, 2020). Therefore, the treatment cost often results in financial catastrophe and distress compelling a household to resort to alternative ways of financing, which pushes the household in a deeper financial debt (WHO, 2010). Owing to lack of finances, knowledge and technological assistance, cancer patients and their caregivers, especially those who belong to the lower socioeconomic group, face significant financial and emotional struggle (Vashistha et al., 2019).

A comprehensive evidence on out-of-pocket and catastrophic health expenditure (CHE) due to cancer is sparse (Kastor and Mohanty, 2018). Previously, the economic impact of cancer was estimated in systematic reviews assessing the overall economic burden of NCDs in Southeast Asia (Rijal et al., 2018) and in India (Mahal et al., 2010). Rijal et al., (2018) searched Medline and Embase to select quantitative studies published between 2000 and 2016 and conducted in Southeast Asia. Mahal et al., (2010) published a comprehensive discussion paper on economic implication of NCDs including cancer in India. In another review conducted by Vashistha et al., (2019), it was observed that the quality of life of caregivers of cancer patients reduces significantly. Quality of life was further 
lowered with increase in duration of care, especially for lower income households. This further brings the need to emphasise studying the economic burden of cancer in order to determine the extent to which mitigation of such a burden is required. Thecurrent systematic review aims to answer the following research questions:

1. What are the pooled estimates for OOPE on inpatient and outpatient care and the proportion of individuals facing $\mathrm{CHE}$ due to the treatment of selected non-communicable disease (cancer) among adults in India?

2. What are the various modes of distressed health financing (DHF) adopted by individuals for the treatment of cancer in India?

3. What are the factors associated with CHE faced due to the treatment of cancer among adults in India?

\section{Materials and Methods}

The current systematic review is registered with PROSPERO (Registration number: CRD42020209497) and is reported following the MOOSE guidelines (Stroup et al, 2000).

\section{Criteria for selection of studies}

All observational studies and government survey reports conducted among Indian adults (more than 18 years old, both males and females) suffering from cancer and published between January 2011 and July 2020 were included in the review. Newsletters, commentaries, editorials, studies conducted among cancer patients less than 18 years old or outside India and published before the year 2011 were excluded from the review.

\section{Search strategy}

Three bibliographic databases (PubMed, Embase, and Scopus) and government websites were searched for relevant studies and reports. Search terms and keywords based on population and exposure and those suggested by advanced database search operators were used to find relevant studies (supplementary Table 1). Boolean operators, such as "AND", "OR" and "NOT" were used for refining the search strategy.

\section{Screening Process}

After removing duplicate studies, titles and abstracts were screened independently by two reviewers using a piloted study screening tool. Subsequently, full-text screening of the selected studies was done and eligible studies were included in the review. Any discrepancies among reviewers were discussed with the third reviewer and resolved through consensus.

\section{Data extraction}

Data extraction was performed by the first and second reviewer independently using a modifiable pre-designed data extraction form and a sample of data was crosschecked by the third reviewer. All OOPE was recorded in the Indian currency (INR) and US dollars (USD) and final expenditure was computed for the year 2020 as baseline. The 'Campbell and Cochrane Economics Methods Group (CCEMG) - Evidence for Policy and Practice Information
(EPPI) - Centre Cost Converter' (v.1.6 last update: 29 April 2019) was used for cost conversions (Shemilt et al., 2019). If the year of measuring OOPE was not mentioned, the year before study publication was taken as the reference year for respective cost conversions. Outcomes, such as proportion of individuals facing $\mathrm{CHE}$, modes of DHF and factors associated with CHE were also recorded.

\section{Risk of bias assessment}

The study quality and risk of bias in the studies was checked using the Appraisal tool for Cross-sectional studies (AXIS) tool, which was modified based on the requirement of the current review (supplementary file) (Dowens et al., 2016). The minimum and maximum score that could be allotted to each study were 0 and 20 , respectively. Studies with a score of 16 or above $(80 \%), 12-15(60-75 \%)$ or and less than $12(<60 \%)$ were considered to be of high, moderate and satisfactory quality, respectively.

\section{Data Analysis}

Narrative synthesis of data was undertaken for all the included studies. $\mathrm{I}^{2}$ test was applied to check heterogeneity among the studies while conducting meta-analysis using the Comprehensive Meta-Analysis software (Higgins et al, 2019). In case of high heterogeneity, random effect model was used as the tau-squared value was non-zero. The pooled estimates were reported along with the $\mathrm{I}^{2}$ values with $95 \%$ confidence interval (CI).

\section{Outcome measures}

The following outcome measures were included in this review:

i. Direct OOPE, which comprised of the consultant charges, charges for diagnostic tests, amount spent on medicines and medical appliances, charges during stay at hospital, food, accommodation and transportation charges.

ii. Indirect OOPE, which included wage loss of the patient and the caretaker

iii. CHE, that is, when OOPE was more than $10 \%$ of total consumption expenditure or $40 \%$ of total non-consumption expenditure of the household.

iv. Modes of DHF, that is, alternative ways a household adopts to pay for cancer treatment.

\section{Results}

After searching all accessible databases, a total of 9607 records were identified. After de-duplication, title and abstracts were screened for 8895 records. Out of these, 61 records were identified for full-text screening. After following strict inclusion and exclusion criteria, 21 studies were found eligible to be included in the systematic review. Two records were identified after manual searching of the references of the selected studies. A total of 23 studies were included and out of these, 15 records were included in the meta-analysis.

Figure 1 shows the steps followed for study selection based on the PRISMA guidelines (Moher et al., 2019). 
Table 1. Overall Characteristics of the Selected Studies

\begin{tabular}{|c|c|}
\hline Characteristics of the studies & $\begin{array}{l}\text { Number of } \\
\text { studies }(\%)\end{array}$ \\
\hline \multicolumn{2}{|l|}{ Year of Publication } \\
\hline $2011-2015$ & $14(63.64 \%)$ \\
\hline 2016-2020 & $8(36.36 \%)$ \\
\hline \multicolumn{2}{|l|}{ Year of cost estimation } \\
\hline $1995-2005$ & $2(9.09 \%)$ \\
\hline $2006-2015$ & $16(72.73 \%)$ \\
\hline $2016-2020$ & $4(18.18 \%)$ \\
\hline \multicolumn{2}{|l|}{ Location (11) } \\
\hline North zone & $7(31.82 \%)$ \\
\hline East zone & $2(9.09 \%)$ \\
\hline West zone & $1(4.54 \%)$ \\
\hline South zone & $3(13.64 \%)$ \\
\hline Pan-India & $9(40.91 \%)$ \\
\hline \multicolumn{2}{|l|}{ Outcomes } \\
\hline Studies on OOPE & $17(77.28 \%)$ \\
\hline Studies on financial catastrophe & $6(27.27 \%)$ \\
\hline Studies on distress financing & $5(22.73 \%)$ \\
\hline \multicolumn{2}{|l|}{ Type of cancer } \\
\hline General (not specified) & $13(59.1 \%)$ \\
\hline Specific (e.g., breast, oral, cervical cancer, etc.) & $9(40.9 \%)$ \\
\hline \multicolumn{2}{|l|}{ Sample size range $(8-11,112)$} \\
\hline $0-500$ & 19 \\
\hline $501-1,000$ & 3 \\
\hline$>1,000$ & 1 \\
\hline
\end{tabular}

Systematic Review on Economic Impact of NCDs in India

\section{Overall study characteristics}

The current systematic review included 23 studies with data related to a total of 17,760 cancer patients. The overall study characteristics are described in Table 1. Figure 2 represents the geographical distribution of included studies.

Characteristics of individual studies included in the systematic review

Out of all studies, $34.8 \%(n=8)$ studies were cross-sectional, $34.8 \%(\mathrm{n}=8)$ were prospective studies and $30.4 \%$ studies were secondary analyses of pan-India cross-sectional survey data. Approximately 52.2\% $(n=12)$ studies were community-based (or analysed data from community-based surveys) while the remaining $47.3 \%(\mathrm{n}=11)$ studies were done in a hospital setting (supplementary Table 2).

Quality and risk of bias assessment of the studies included in the systematic review

Out of the selected 23 articles, $43.48 \%$ articles were found to be of high quality, $47.83 \%$ of moderate quality and $8.69 \%$ of satisfactory quality (supplementary table $3)$. Approximately $43.48 \%$ of the studies $(n=10)$ had a high risk of selection bias, $60 \%(n=9)$ to had high risk of non-response bias and $13.04 \%(n=3)$ had a high risk of information bias (supplementary Table 4, Figure 3). Among all, 82.6\% studies used validated tools to measure outcomes and risk factors.

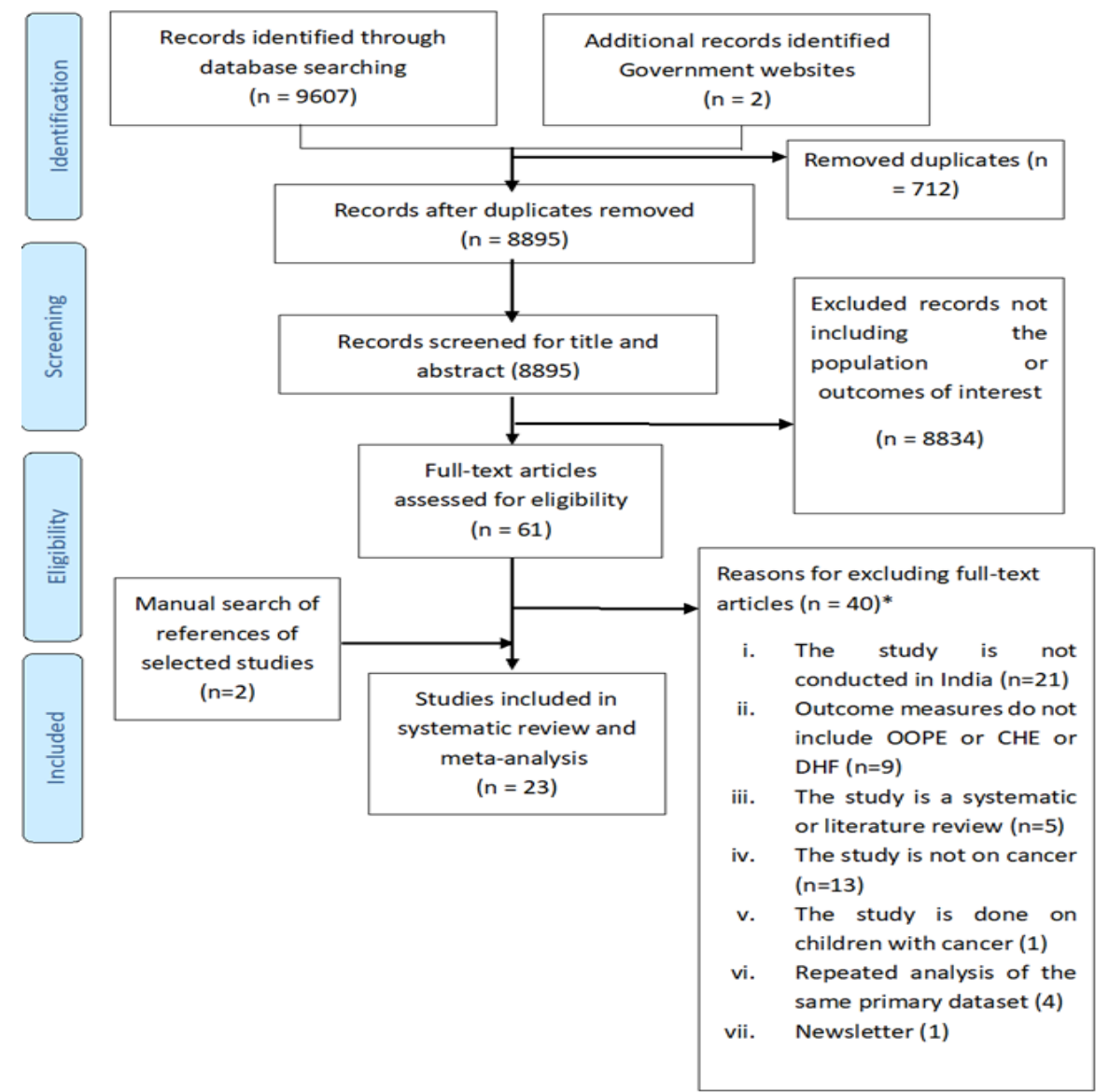

*Some of the studies had multiple reasons for being excluded from the review

Figure 1. Prisma Flow Diagram for Selection of Eligible Studies 


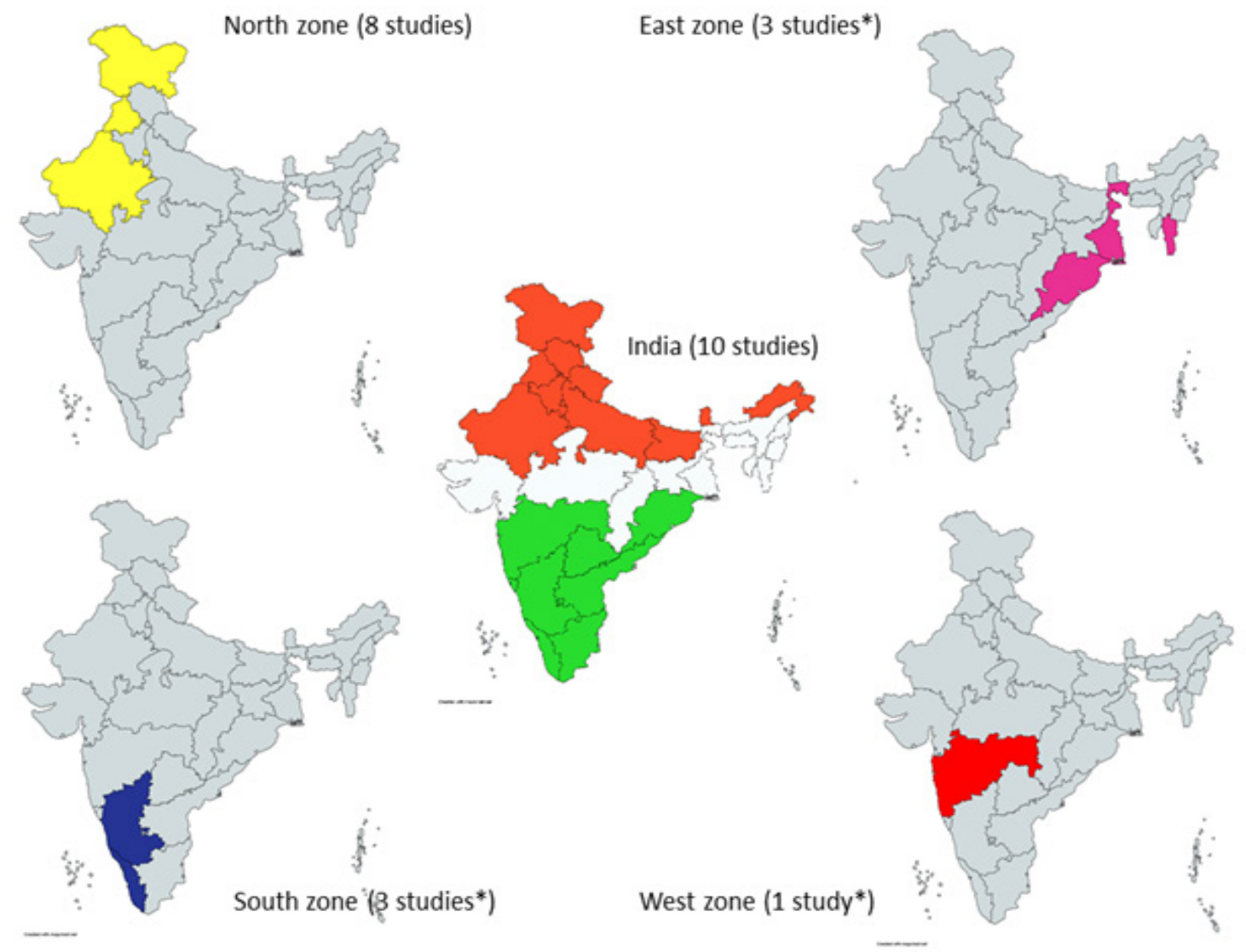

Figure 2. Geographical Distribution of Studies Included in the Review. *One of the studies was conducted in all the three zones. The maps shown above were created using a web-based tool, mapcharts.(16) They do not indicate the political administrative boundaries of India and are only for representation purposes. Zonal division is done as per the Zonal Councils of India.

OOPE incurred on cancer treatment in India

A total of $12(52.2 \%)$ studies measured direct and five out of these ( $41.6 \%$ of 12 studies) measured indirect OOPE on cancer treatment (supplementary Table 5).

The reference period for estimating direct OOPE on inpatient cancer care was 365 days. The pooled mean for direct OOPE on inpatient cancer care was found to be 83396.07 INR (4405.96 USD) (95\% $\mathrm{CI}=44591.05-122202.0$ ) (Figure 4) with a high-level between-study heterogeneity $\left(\mathrm{I}^{2}=99.98, \mathrm{p}=0.0001\right)$. Among the selected studies, five reported direct OOPE on outpatient cancer care taking 15 days as reference

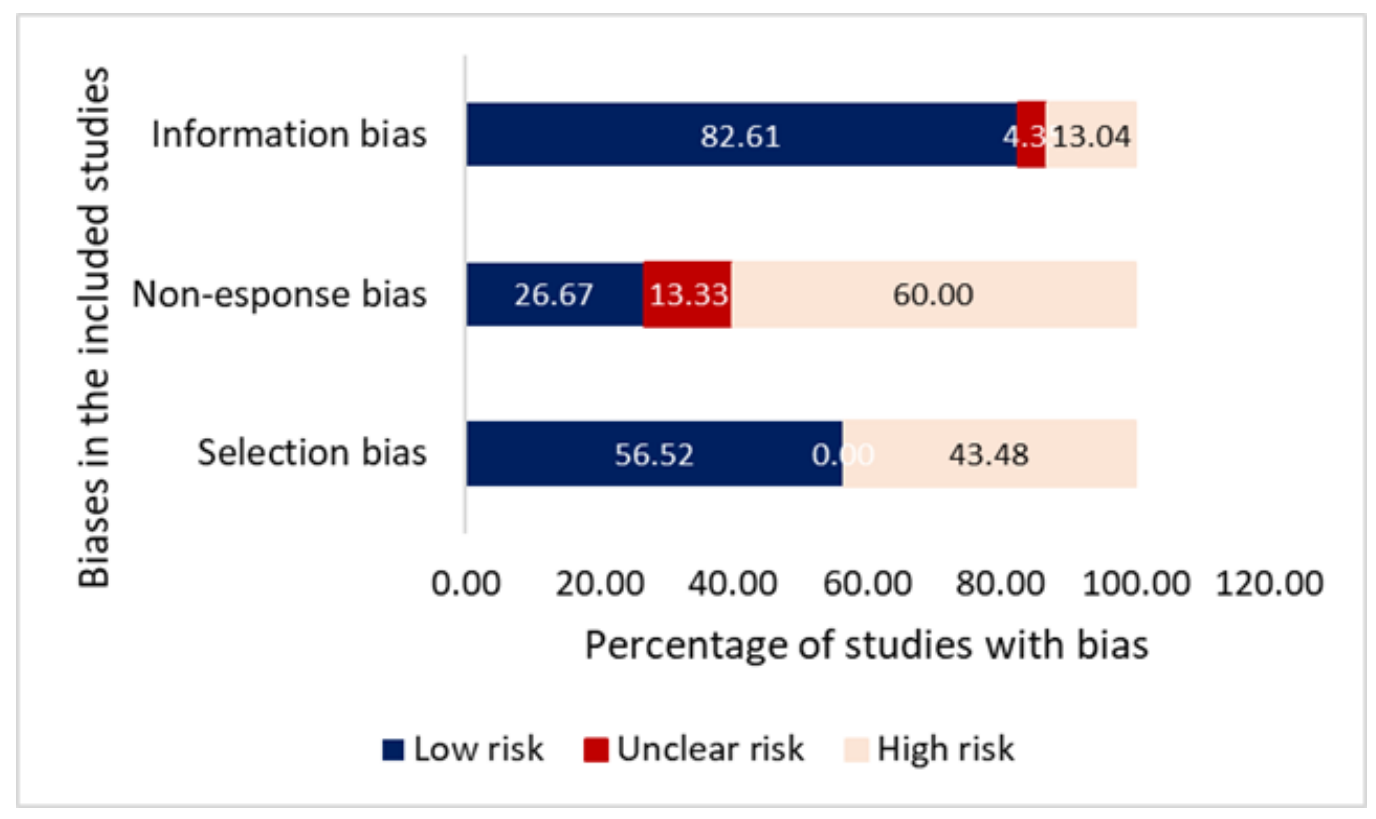

Figure 3. Percentage of Included Studies with Different Risks of Bias 
Study name

NSSO, 2020

Kastor et al, 2018

Kaur et al, 2017

Joseph et al, 2016

Goyal et al, 2018

Wani et al, 2013

Mahal et al, 2013

Summary

effect

$-375000.00$
Mean and $95 \% \mathrm{Cl}$

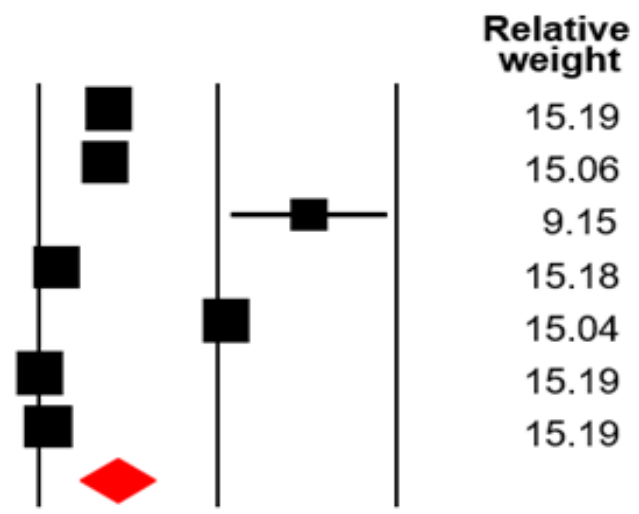

$\begin{array}{lll}0.00 & 187500.00 \quad 375000.00\end{array}$

Figure 4. Meta-Analysis Results for Direct OOPE on Inpatient Cancer Care: Random Effect Model

period. The pooled mean direct OOPE on outpatient cancer care was found to be 2653.12 INR (140.17 USD) (95\% CI=-251.28-5557.53; $\mathrm{SE}=1481.56 \mathrm{INR} ; \mathrm{I}^{2}=99.94$, $\mathrm{p}=0.0001)$ (Figure 5). The pooled mean total direct OOPE on both inpatient and outpatient cancer care was 47138.95 INR (2490.43 USD) (95\% CI=37589.43-56690.74; $\mathrm{SE}=4872.88 \mathrm{INR} ; \mathrm{I}^{2}=96.88, \mathrm{p}=0.0001$ ) (Figure 6).

Only five of the included studies reported indirect cost incurred on cancer care and its pooled estimate was found to be 11908.50 INR (629.15 USD) $(95 \% \mathrm{CI}=-5909.33$ 29726.31; $\mathrm{SE}=9090.89$ INR; I2=99.84; $\mathrm{p}=0.0001$ ) (Figure 7).

Catastrophic health expenditure (CHE) on cancer treatment in India

The reported proportion of cancer patients suffering financial catastrophe varied across the studies (supplementary Table 6). The pooled event rate of individuals facing catastrophic health expenditure availing cancer care was found to be $0.627\left(62.7 \% ; \mathrm{I}^{2}=98.60 ; \mathrm{p}\right.$ $=0.0001)$ (Figure 8).

Modes of distress financing for cancer treatment in India Table 2 describes the modes of DHF adopted by cancer patients while undergoing treatment. The findings reveal that savings or income, borrowing money from friends or social connections, and selling property or assets are most commonly adopted modes. One of the studies also revealed discontinuing treatment and continuing to live with cancer as one of the coping strategies.

Correlates of Catastrophic health expenditure on cancer care in India

Out of the six studies addressing CHE, five had

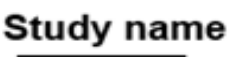

NSSO, 2020

Kaur et al, 2017

Mahal et al, 2013

Summary effect

$-125000.00-62500.00$

\section{Mean and $95 \% \mathrm{Cl}$}
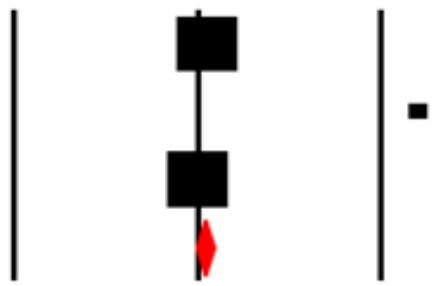

\section{Relative weight}

49.40

1.22

49.38

Figure 5. Meta-Analysis Results for Direct OOPE on Outpatient Care: Random Effect Model 
Table 2. Result of Meta-Analysis for Estimating OOPE and CHE

\begin{tabular}{|c|c|c|c|c|c|}
\hline \multicolumn{6}{|c|}{ Direct OOPE on inpatient cancer care in India } \\
\hline Study Name & Mean & Standard Error & Variance & Lower limit & Upper limit \\
\hline NSSO, 2020 & 75689.09 & 2.19 & 4.82 & 75684.8 & 75693.39 \\
\hline Kastor et al, 2018 & 70763.17 & 4778.85 & 222837415.4 & 61396.79 & 80129.55 \\
\hline Kaur et al, 2017 & 284688.06 & 41253.02 & 1701811739 & 203833.63 & 365542.5 \\
\hline Joseph et al, 2016 & 19925.98 & 946.59 & 896038.77 & 18070.69 & 21781.27 \\
\hline Goyal et al, 2014 & 198198.58 & 5063.85 & 25642546.44 & 188273.62 & 208123.54 \\
\hline Wani et al, 2013 & 3430.85 & 75.48 & 5697.83 & 3282.9 & 3578.8 \\
\hline Mahal et al, 2013 & 12061.32 & 365.78 & 133792.6 & 11344.41 & 12778.23 \\
\hline Summary effect & 83396.52 & 19799.08 & 392003454.3 & 44591.05 & 122202 \\
\hline \multicolumn{6}{|c|}{ Direct OOPE on outpatient cancer care in India } \\
\hline Study Name & Mean & Standard Error & Variance & Lower limit & Upper limit \\
\hline NSSO, 2020 & 3238.92 & 4.75 & 22.56 & 3229.61 & 3248.23 \\
\hline Kaur et al, 2017 & 75282.16 & 13235.45 & 175177246.8 & 49341.15 & 101223.17 \\
\hline Mahal et al, 2013 & 268.73 & 49.38 & 2438.27 & 171.95 & 365.51 \\
\hline Summary effect & 2653.13 & 1481.86 & 2195924.37 & -251.28 & 555.53 \\
\hline \multicolumn{6}{|c|}{ Total direct OOPE on cancer care in India } \\
\hline Study Name & Mean & Standard Error & Variance & Lower limit & Upper limit \\
\hline NSSO, 2020 & 56105.95 & 1.99 & 3.98 & 56102.04 & 56109.86 \\
\hline Dinesh et al, 2019 & 37242.05 & 4401.8 & 19375851.15 & 28614.68 & 45869.42 \\
\hline Chauhan et al, 2019 & 45749.05 & 1531.11 & 2344294.11 & 42748.63 & 48750.47 \\
\hline Summary effect & 47140.08 & 4872.88 & 23744905.93 & 37589.43 & 56690.74 \\
\hline \multicolumn{6}{|c|}{ Indirect OOPE on cancer care in India } \\
\hline Study Name & Mean & Standard Error & Variance & Lower limit & Upper limit \\
\hline Dinesh et al, 2019 & 2802.88 & 733.34 & 537790.49 & 1365.56 & 4240.2 \\
\hline Chauhan et al, 2019 & 20984.69 & 53.32 & 2843.39 & 20880.18 & 21089.2 \\
\hline Summary effect & 11908.5 & 9090.89 & 82644337.3 & -5909.33 & 29726.31 \\
\hline \multicolumn{6}{|c|}{ Proportion of cancer patients facing CHE in India } \\
\hline Study name & Event rate & & Lower Limit & & Upper Limit \\
\hline Sangar et al, 2019 & 0.3 & & 0.292 & & 0.309 \\
\hline Chauhan et al, 2019 & 0.34 & & 0.296 & & 0.387 \\
\hline Kastor et al, 2018 & 0.79 & & & & 0.831 \\
\hline Basavaiah et al, 2018 & & & 0.671 & & 0.839 \\
\hline Jain et al, 2016 & 0.84 & & 0.786 & & 0.883 \\
\hline Summary effect & 0.627 & & 0.378 & & 0.823 \\
\hline
\end{tabular}

analysed its predictors which were not comparable. Therefore, meta-analysis to determine the effect of various factors associated with CHE could not be conducted.

Sangar et al., (2019) found that headcount for CHE was more among richer sections as compared to the poorer ones. Chauhan et al., (2018) found that the odds of CHE were higher for lower income quartile patients (OR: 5.6, 95\% CI: 2.6-12.4, p-value: $<0.001$ ) than those in the highest income quartile. Also, the patients undergoing intensity-modulated radiotherapy (IMRT) faced higher odds of incurring $\mathrm{CHE}(\mathrm{OR}=3.516$; $95 \% \mathrm{CI}=1.61-7.66$, $\mathrm{p}$-value $<0.002$ ) as compared to those receiving two-dimensional radiotherapy (2-DRT). Rajpal et al., (2018) found in their study that $36.3 \%$ (public healthcare facilities) and $33.7 \%$ (private healthcare facilities) of cancer patients were spending more than $10 \%$ of their annual per capita household expenditure (PCHE).
Over $50 \%$ of the cancer patients from low-income households were reported to be spending more than $10 \%$ and $20 \%$ percent of PCHE. In contrast, around $26 \%$ of richer households were reported spending more than $10 \%$ and $20 \%$ of their annual income on cancer treatment.

In another study by Jain and Mukherjee (2016), lower income households were reported to be 39.38 times and middle income households to be 5.79 times more likely to face CHE as compared to higher income households. Cancer patients utilising private facilities were reported to be 62.2 times more likely to face CHE than those seeking treatment at public healthcare facilities. Also, patients with second or higher stage of cancer were reported to be at 16.29 times higher risk of facing CHE (Jain and Mukherjee, 2016). Tripathy et al., (2016) found that the odds of facing CHE were 12.2 times for cancer treatment as compared to that for a communicable disease. 
Table 3. Modes of Distress Financing and Coping Strategies for High OOP Payments on Cancer Care in India

\begin{tabular}{lll}
\hline Reference article & Reported modes of distress financing & Proportion of cancer patients \\
\hline Alexander et al, & Savings & $28 \%$ \\
2019 & Mortgaging or selling assets & $30 \%$ \\
& High-interest loan or discontinuation of therapy & $13 \%$ \\
& Discontinued treatment & $3 \%$ \\
Jain and Mukherjee,, & Borrowed money at low interest (0-15\% p.a.) rates & $84.60 \%$ \\
2016 & Used social nets that is monetary assistance form relatives and friends & $74.70 \%$ \\
& Savings & $74.20 \%$ \\
& Used financial assets that is shares, mutual funds and gold & $53.40 \%$ \\
& Delayed payments of pre-existing loans & $48.90 \%$ \\
& Sold economic productive assets & $41.20 \%$ \\
& Renting out & $33 \%$ \\
& Delayed payment of bills & $19.90 \%$ \\
& Pawned jewellery & $16.70 \%$ \\
& Borrowed money at a high interest ( $\geq 15 \%)$ rate & $15.80 \%$ \\
took credit from local shop (15.8\%), & $15.80 \%$ \\
Joe, 2015 & financing by aid (govt/private) (14\%) & $14 \%$ \\
& Income/savings & - \\
& Borrowing & \\
& Contributions from relatives or friends & \\
\hline & sale of assets & \\
\hline
\end{tabular}

Study name

NSSO, 2020

Dinesh, 2019

Chauhan, 2019

Summary effect

\section{Mean and $95 \% \mathrm{Cl}$}

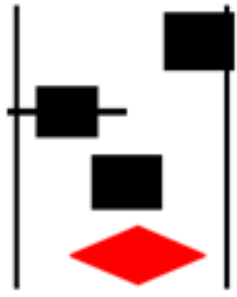

Relative weight

36.56

28.16

35.28

\section{$\begin{array}{lllll}-60000.00 & -30000.00 & 0.00 & 30000.00 & 60000.00\end{array}$}

Figure 6. Meta-Analysis Results for Total Direct OOPE on Inpatient and Outpatient Care: Random Effect Model

Study name

Dinesh et al, 2019
Chauhan et al, 2019
Summary Effect

Mean and $95 \% \mathrm{Cl}$

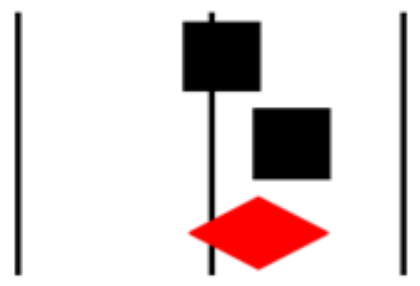

\section{$\begin{array}{lllll}-100000.00 & -50000.00 & 0.00 & 50000.00 & 100000.00\end{array}$}

Figure 7. Meta-Analysis Results for Indirect OOPE in Cancer Care: Random Effect Model 


\section{Study name}

Sangar et al, 2019

Chauhan et al, 2019

Kastor et al, 2018

Basavaiah et al, 2018

Jain et al, 2016

Summary effect
Event rate and $95 \% \mathrm{Cl}$

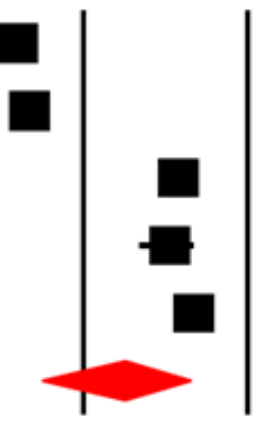

Relative

weight

20.35

20.19

20.07

19.52

19.86

$\begin{array}{lllll}-1.00 & -0.50 & 0.00 & 0.50 & 1.00\end{array}$

Figure 8. Meta-Analysis Results for Proportion of Cancer Patients Facing CHE: Random Effect Model

\section{Discussion}

The current systematic review included 23 eligible studies with an overall picture of OOPE incurred, CHE faced and modes of distress financing adopted by cancer patients in India. The studies varied in design, sampling strategy, and reporting of outcome measures rendering themselves at risk of including different types of biases. Selection bias was the most commonly encountered bias among studies followed by non-response bias. Information on response rate, reporting standard deviation or confidence intervals and median costs was lacking in some of the studies given the fact that economic studies often show skewed distribution. In the current systematic review where studies showed highly variable sample sizes, a normal distribution of outcome measures was less likely. Moreover, the studies also vary in quality due to inconsistent definitions and lack of reference group.

The pooled mean direct cost for inpatient cancer treatment is significantly high (83345.026 INR; $p=0.0001)$ as compared to other costs. A similar finding was also reported in a systematic review conducted by Rijal et al., (2018). Another systematic review on NCDs (including cancer) also reported that the highest proportion of OOPE is attributable to direct medical cost, especially medications, for the treatment of cancer. The pooled mean OOPE on outpatient cancer care in a reference period of 15 days was found to be 2653.12 (1481.87) INR.

Through these findings, it is evident that the OOPE on cancer care is high and unaffordable, which is also reported by all of the included studies. However, individual study cost estimates were highly variable and heterogeneity was evident from the meta-analysis results. One of the possible reasons could be attributed to the differences in study designs. Cross-sectional studies record the costs at one point of time while prospective studies give an elaborate picture. Those with large sample size are likely to report more precise outcome measures as compared to small-scale studies with non-representative sample population. Another reason could be the variation in operational definitions of direct OOPE on cancer care and type of healthcare facility utilised resulting in significantly different results.

Very few studies measured indirect cost associated with cancer treatment. The pooled mean indirect OOPE was also very high [11908.53 (9090.89) INR], however, the result was not statistically significant $(p=0.190)$. It is evident that indirect expenditure in the form of wage loss of the patient and the caregiver is high. The employment sector being largely informal, health seeking behaviour may also be guided by loss of wages on daily basis due to cancer treatment. Inconsistencies in defining indirect OOPE among the included studies was also seen across these studies.

The pooled estimate of the percentage of cancer patients facing CHE was $62.7 \%$, which is drastically high. The reason for such a high proportion of cancer patients experiencing CHE may be attributed to different cut-off levels to determine CHE across studies, differences in incomes and socioeconomic status of cancer patients, cost of public and private healthcare facilities, cost of various treatment modalities and advancing stage of cancer (Rajpal et al., 2-18; Jain and Mukherjee, 2018). The possibility of poor sections of the society to be financially drained and henceforth, not seeking medical care is also high. This was evident from the study conducted in Karnataka (Alexander et al., 2019) which reported 3\% of the cancer patients who had discontinued treatment due to high OOPE and associated financial catastrophe.

The most common modes of DHF for cancer care are savings, borrowing money and sale of assets. Similar findings have been reported by a previously done systematic review (Rijal et al., 2018). The most dreadful coping strategy was discontinuing treatment. Health insurance being an unpopular practice in India, the dependency on alternative means of financing makes the households unable to cope with similar health-related incidents in future. After more than one year of the 
launch of Ayushman Bharat under the Pradhan Mantri Jan Aarogya Yojana, no study has been conducted so far to evaluate the current status of healthcare financing in cancer, especially among the vulnerable families who are beneficiaries of the program.

Only a few out of the selected 23 studies have identified the possible predictors of CHE among cancer patients. Such a gap in existing data may be due to poorly functioning healthcare management systems and absence of population-based cancer registries in India. This results in underreporting of cancer cases and underestimation of overall economic impact of cancer.

To monitor the level of financial protection, WHO recommends using incidence of $\mathrm{CHE}$ among patients seeking health care services. Most of the studies included in this review addressed the proportion of individuals facing $\mathrm{CHE}$ at one point of time. In addition, half of the studies were secondary analyses of cross-sectional surveys, failing to provide evidence on temporal relationship between $\mathrm{CHE}$ and its predictors.

As per the findings of current systematic review, only a handful of primary studies have been conducted on the economics of cancer in the country, especially when available literature provides strong evidence that cancer has the highest economic burden among all NCDs, both on cancer patients and health systems. The current knowledge gap of its impact subsequently slows down the already lagging preventive and curative health program implementation in the target populations to help financially challenged households. Hence, crucial information still lies in the hands of future robust research studies. Emphasising on the duration for which a household is under financial catastrophe is also important, especially in case of cancer where the treatment cost is high and the disease is often associated with recurrences and complications.

The main limitation of our review is using only three databases for screening and identifying studies published after 2010. However, a thorough search of the references was also done to include any eligible studies in the review. Also, in most of the studies, the status of cancer was selfreported and only a few studies used diagnosed cancer as the inclusion criteria, rendering the current analysis to have possibly overestimated the costs. Besides this, recall bias in providing cost-related information in all the studies is another challenge that may have resulted in under-estimation of actual OOPE on cancer treatment after data pooling. Due to lack of availability of data on correlates of financial catastrophe in the included studies, the current review could not identify the risk factors or predictors associated with it.

One of the strengths of the current systematic review is the use of a comprehensive, peer-reviewed and validated tool to assess the quality as well as risk of bias of the included studies. The review also provides pooled estimates in both Indian and US currencies allowing to extrapolate the pooled cost estimates to the current year (2020) and increase comparability with international studies. It was able to highlight the seriousness of expenditures incurred by cancer patients (both direct and indirect cost) while seeking cancer treatment. The costs are unaffordable for
Systematic Review on Economic Impact of NCDs in India

people already existing below the poverty line. This brings the need of special income- or treatment-related policy and evidence-informed nationally tailored prepayment mechanisms with consideration of patients suffering from cancer, especially for those from the informal sector and low socioeconomic status. Through the current systematic review, the gaps in data availability, poor data reporting and high variability among studies assessing the cost could also be highlighted. These findings reveal the need of robust research required in adequately measuring costrelated data with reference/comparator group along with optimal study design and universal definitions to measure outcomes to prevent variations in cost estimates. It also emphasises on the need of preventive cancer strategies and early detection of cancer. A cancer registry to record all cost-related data could be incorporated in the present healthcare system to identify individuals likely to face financial distress to whom subsidised care could be provided. An interventionist mass insurance policy as a joint venture between the government and private insurance companies for cost sharing can potentially make cancer care affordable.

\section{Author Contribution Statement}

The authors confirm contribution to the paper as follows:

Study conception and design: Anushikha Dhankhar, Ranjeeta Kumari; data collection: Anushikha Dhankhar, Yogesh A Bahurupi; analysis and interpretation of results: Anushikha Dhankhar, Ranjeeta Kumari, Yogesh A Bahurupi; draft manuscript preparation: Anushikha Dhankhar. All authors reviewed the results and approved the final version of the manuscript.

\section{Acknowledgements}

\section{Statement conflict of Interest} None.

\section{References}

Alexander A, Kaluve R, Prabhu JS, et al (2019). The impact of breast cancer on the patient and the family in Indian perspective. Indian J Palliat Care, 25, 66.

Basavaiah G, Rent PD, Rent EG, et al (2018). Financial impact of complex cancer surgery in India: A study of pancreatic cancer. J Glob Oncol, 4, 1-9.

Batra A, Gupta I, Mukhopadhyay A (2014). Does discrimination drive gender differences in health expenditure on adults: evidence from Cancer patients in rural India. Indian Statistical Institute, New Delhi, India.

Central Bureau of Health Intelligence (CBHI) (2019). National Health Profile, 14th issue.pdf. [Internet]. [cited 2020 July 12]. Available from: https://cbhidghs.gov.in/showfile. php?lid=1147.

Chauhan AS, Prinja S, Ghoshal S, Verma R (2019). Economic burden of head and neck cancer treatment in North India. Asian Pac J Cancer Prev, 20, 403.

Dinesh TA, Nair P, Abhijath V, Jha V, Aarthy K (2020). Economics of cancer care: A community-based crosssectional study in Kerala, India. South Asian J Cancer, 9, 7.

Downes MJ, Brennan ML, Williams HC, Dean RS (2016).

Asian Pacific Journal of Cancer Prevention, Vol 22

679 
Development of a critical appraisal tool to assess the quality of cross-sectional studies (AXIS). BMJ Open, 6, e011458.

Higgins JP, Thomas J, Chandler J, et al (2019). Cochrane handbook for systematic reviews of interventions. John Wiley \& Sons.

Government of India, NSSO (2020). Ministry of Statistics and Programme Implementation. Health in India: NSS 75th round.pdf. [cited 2020 September 3]. [Internet]. http://mospi.nic.in/sites/default/files/publication_reports/ KI_Health_75th_Final.pdf.

Government of India, NSSO (2014). Ministry of Statistics and Programme Implementation. Health in India: NSS 71st round.pdf. [cited 2020 September 3]. [Internet].

Government of India, Ministry of Home Affairs (2011). Compositions of Zonal Councils of India. [Internet]. [cited: 2020 August 16]. Available from: https://www.mha.gov.in/ zonal-council.

Goyal S, Tiwari VK, Nair KS, Raj S (2014). Risk factors and costs of oral cancer in a tertiary care hospital in Delhi. Asian Pac J Cancer Prev, 15, 1659-65.

Jain M, Mukherjee K (2016). Economic burden of breast cancer to the households in Punjab, India. Int J Med Public Health, 6.

Joe W (2015). Distressed financing of household out-of-pocket health care payments in India: incidence and correlates. Health Policy Plan, 30, 728-41.

Joseph N, Gupta S (2016). Assessment of economic impact among in-patients with non-communicable diseases in a private tertiary care Hospital in Southern India. J Clin Diagn Res, 10, LM04.

Karan A, Engelgau M, Mahal A (2010). The economic implications of non-communicable disease for India. The World Bank.

Kastor A, Mohanty SK (2018). Disease and age pattern of hospitalisation and associated costs in India: 1995-2014. BMJ Open, 8, e016990.

Kastor A, Mohanty SK (2018). Disease-specific out-of-pocket and catastrophic health expenditure on hospitalization in India: Do Indian households face distress health financing? PLoS One, 13, e0196106.

Kaur G, Prinja S, Malhotra P, et al (2018). Cost of treatment of multiple myeloma in a public sector tertiary Care Hospital of North India. Indian J Hematol Blood Transfus, 34, 25-31.

Mahal A, Karan A, Fan VY, Engelgau M (2013). The economic burden of cancers on Indian households. PLoS One, 8 , e71853.

Mukhopadhyay A, Das S, Sharma K (2011). Estimating the Economic Burden of Cancer at a Tertiary Public Hospital: A Study at the All India Institute of Medical Sciences. Research Gate, New Delhi, India.

Minas. Mapchart.net. [Internet]. [cited: 2020 August 16]. Available from: https://mapchart.net/india.html

Nair KS, Raj S, Tiwari VK, Piang LK (2013). Cost of treatment for cancer: experiences of patients in public hospitals in India. Asian Pac J Cancer Prev, 14, 5049-4.

Nethan S, Sinha D, Mehrotra R (2017). Non communicable disease risk factors and their trends in India. Asian Pac J Cancer Prev, 18, 2005.

Pakseresht S, Ingle GK, Garg S, Singh MM (2011). Expenditure audit of women with breast cancer in a tertiary care hospital of Delhi. Indian J Cancer, 48, 428.

Rajpal S, Kumar A, Joe W (2018). Economic burden of cancer in India: Evidence from cross-sectional nationally representative household survey, 2014. PLoS One, 13, $\mathrm{e} 0193320$.

Rijal A, Adhikari TB, Khan JA, Berg-Beckhoff G (2018). The economic impact of non-communicable diseases among households in South Asia and their coping strategy: A systematic review. PLoS One,13, e205745.

Sangar S, Dutt V, Thakur R (2019). Comparative assessment of economic burden of disease in relation to out of pocket expenditure. Front Public Health, 7, 9.

Selvaraj S, Farooqui HH, Karan A (2018). Quantifying the financial burden of households' out-of-pocket payments on medicines in India: a repeated cross-sectional analysis of National Sample Survey data, 1994-2014. BMJ Open, 8.

Shemilt I. CCEMG-EPPI-Centre Cost Converter; Version 1.4. 2019. The Campbell and Cochrane Economics Methods Group (CCEMG) and the Evidence for Policy and Practice Information and Coordinating Centre (EPPI-Centre).

Stroup DF, Berlin JA, Morton SC, Olkin I, Williamson GD (2000). MOOSE guidelines for meta-analyses and systematic reviews of observational studies. JAMA, 283, 2008-12.

Tripathy JP, Prasad BM, Shewade HD, et al (2016). Cost of hospitalisation for non-communicable diseases in India: are we pro-poor?. Trop Med Int Health, 21, 1019-28.

Vashistha V, Poulose R, Choudhari C, Kaur S, Mohan A (2019). Quality of life among caregivers of lower-income cancer patients: A Single-Institutional Experience in India and Comprehensive Literature Review. Asian Pac J Cancer Care, 4, 87-93.

Wani MA, Tabish SA, Jan FA, et al (2013). Cost analysis of in-patient cancer chemotherapy at a tertiary care hospital. J Cancer Res Ther, 9, 397.

World Health Organisation (2018). Noncommunicable diseases [Internet]. [cited 2019 Jun 25]. Available from: https://www. who.int/news-room/fact-sheets/detail/noncommunicablediseases.

World Health Organisation (2010). The world health report: Financing for universal coverage.pdf [Internet]. [cited 2019 Jun 25]. Available from: https://www.who.int/whr/2010/en/

World Health Organisation (2019). Cancer. [Internet]. [cited 2020 July 12]. Available from: https://www.who.int/healthtopics/cancer\#tab=tab_1.

World Health Organisation (2018). WHO-NMH-NVI-18.8-eng. pdf [Internet]. [cited 2019 Jun 25]. Available from: https:// apps.who.int/iris/bitstream/handle/10665/272534/WHONMH-NVI-18.8-eng.pdf?ua=1.

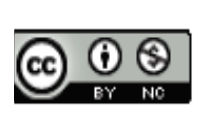

This work is licensed under a Creative Commons AttributionNon Commercial 4.0 International License. 